\title{
Supplementary Information: Linear
}

\section{Combination of Atomic Dipoles to Calculate the Bond and Molecular Dipole Moments of} Molecules and Molecular Liquids

\author{
Kaixuan Chen ${ }^{1-3}$, Wan-Lu Li ${ }^{1-3}$, Teresa Head-Gordon ${ }^{1-5 *}$ \\ ${ }^{1}$ Chemical Sciences Division, Lawrence Berkeley National Laboratory, ${ }^{2}$ Kenneth S. Pitzer Center \\ for Theoretical Chemistry, ${ }^{3}$ Department of Chemistry, ${ }^{4}$ Department of Chemical and \\ Biomolecular Engineering, ${ }^{5}$ Department of Bioengineering, University of California, Berkeley, \\ Berkeley, California 94720, USA \\ E-mail: thg@berkeley.edu
}


Table S1: The bond dipole moments and their sum to define the molecular dipole calculated using DDAPC atomic charges and charge density (Eq. (4) in main text) compared to experiment. One should note that the choice of atomic charge has a significant influence on the value of dipole moment, ${ }^{1,2}$ and in this work we adopt the density derived atomic point charges (DDAPC) produced from $\mathrm{CP} 2 \mathrm{~K}$ for illustrative purposes only. The unit is Debye.

\begin{tabular}{|c|c|c|c|c|c|c|}
\hline \multirow{2}{*}{ Molecule } & \multirow{2}{*}{ Bond } & \multicolumn{2}{|c|}{$\vec{\mu}_{b}$} & \multicolumn{2}{|c|}{$\sum \vec{\mu}_{b}$} & \multirow{2}{*}{$\vec{\mu}_{m}(\exp )}$. \\
\hline & & point charges & charge density & point charges & charge density & \\
\hline \multirow{3}{*}{$\mathrm{CH}_{3} \mathrm{OH}$} & $\mathrm{C}-\mathrm{H}^{a}$ & 0.6 & 1.98 & \multirow{3}{*}{2.64} & \multirow{3}{*}{2.85} & \multirow{3}{*}{1.70} \\
\hline & $\mathrm{C}-\mathrm{O}$ & 3.34 & 3.00 & & & \\
\hline & $\mathrm{O}-\mathrm{H}$ & 1.64 & 1.78 & & & \\
\hline $\mathrm{CH}_{4}$ & $\mathrm{C}-\mathrm{H}^{a}$ & 0.52 & 0.17 & 0.00 & 0.62 & 0.00 \\
\hline $\mathrm{CO}_{2}$ & $\mathrm{C}-\mathrm{O}$ & 3.23 & - & 0.00 & - & 0.00 \\
\hline \multirow{2}{*}{$\mathrm{H}_{2} \mathrm{CO}$} & $\mathrm{C}-\mathrm{O}$ & 2.58 & 1.88 & \multirow{2}{*}{2.16} & \multirow{2}{*}{2.04} & \multirow{2}{*}{2.33} \\
\hline & $\mathrm{C}-\mathrm{H}^{a}$ & 0.39 & 1.96 & & & \\
\hline $\mathrm{H}_{2} \mathrm{O}$ & $\mathrm{O}-\mathrm{H}^{a}$ & 1.45 & 1.87 & 1.82 & 1.98 & 1.85 \\
\hline $\mathrm{H}_{2} \mathrm{O}_{2}$ & $\mathrm{O}-\mathrm{H}^{a}$ & 1.59 & 1.84 & 1.62 & 1.82 & 1.77 \\
\hline \multirow{2}{*}{$\mathrm{HCN}$} & $\mathrm{C}-\mathrm{H}$ & 0.51 & 1.45 & \multirow{2}{*}{2.07} & \multirow{2}{*}{0.32} & \multirow{2}{*}{2.98} \\
\hline & $\mathrm{C}-\mathrm{N}$ & 1.56 & 1.76 & & & \\
\hline \multirow{2}{*}{$\mathrm{HNC}$} & $\mathrm{N}-\mathrm{H}$ & 0.85 & 0.71 & \multirow{2}{*}{1.77} & \multirow{2}{*}{1.08} & \multirow{2}{*}{3.05} \\
\hline & $\mathrm{C}-\mathrm{N}$ & 0.92 & 0.45 & & & \\
\hline \multirow{2}{*}{ HNO } & $\mathrm{O}-\mathrm{N}$ & 0.24 & 0.81 & \multirow{2}{*}{1.16} & \multirow{2}{*}{2.83} & \multirow{2}{*}{1.67} \\
\hline & $\mathrm{N}-\mathrm{H}$ & 1.22 & 2.06 & & & \\
\hline $\mathrm{NF}_{3}$ & $\mathrm{~N}-\mathrm{F}^{a}$ & 0.24 & 3.36 & 0.33 & 1.63 & 0.23 \\
\hline $\mathrm{NH}_{3}$ & $\mathrm{~N}-\mathrm{H}^{a}$ & 1.36 & 1.87 & 1.68 & 4.04 & 1.47 \\
\hline $\mathrm{OF}_{2}$ & $\mathrm{O}-\mathrm{F}^{a}$ & 0.22 & 1.34 & 0.28 & 0.36 & 0.30 \\
\hline MAD & & & & 0.36 & 1.01 & \\
\hline
\end{tabular}

${ }^{a}$ The average of the calculated dipole moments for all same-type bonds within an molecule is used. For example, the three $\mathrm{C}-\mathrm{H}$ bonds within a $\mathrm{CH}_{3} \mathrm{OH}$ molecule. 


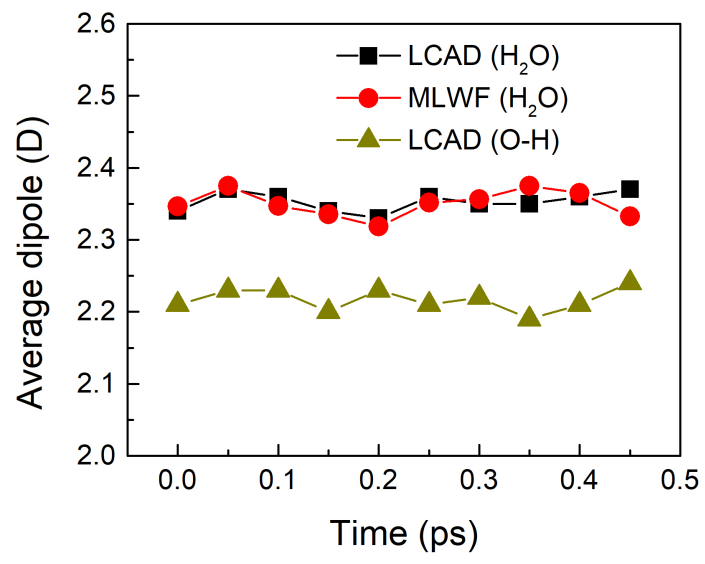

Figure S1: The average $\mathrm{H}_{2} \mathrm{O}$ molecular dipole and $\mathrm{O}-\mathrm{H}$ bond dipole calculated of 10 AIMD snapshots, chosen after it reaches thermal and energetic equilibrium at $1 \mathrm{ps}$. 


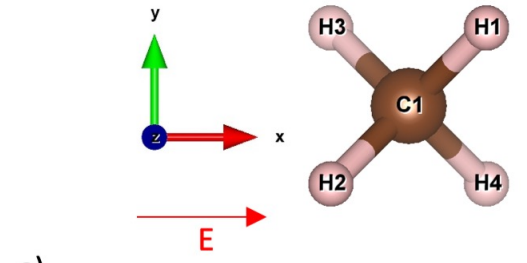

a)
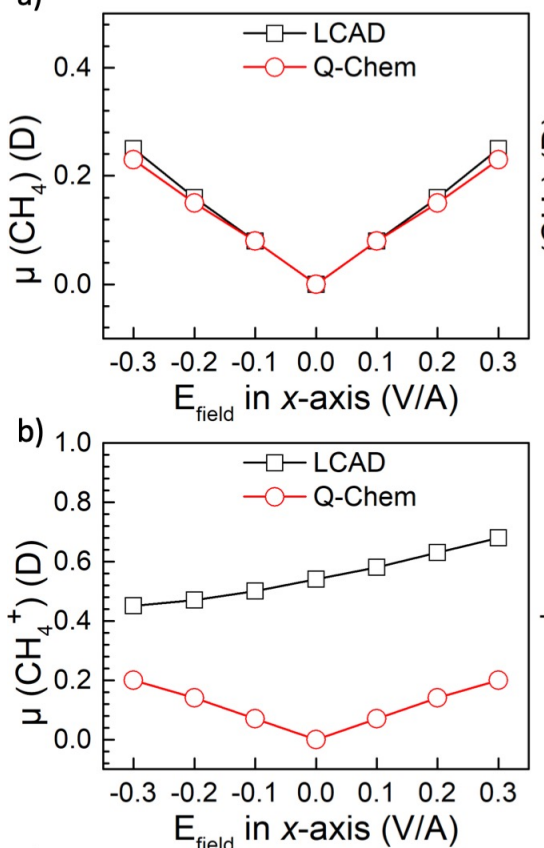

c)

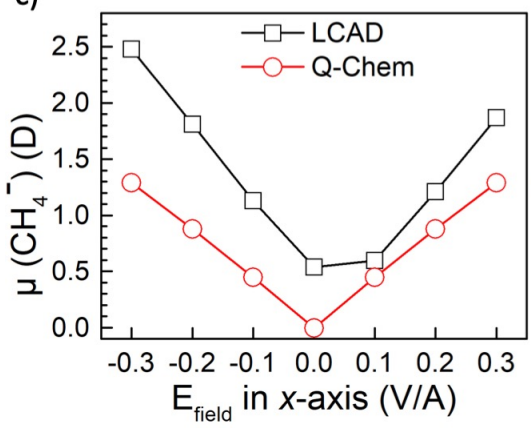

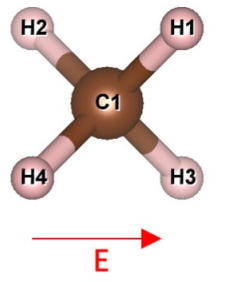
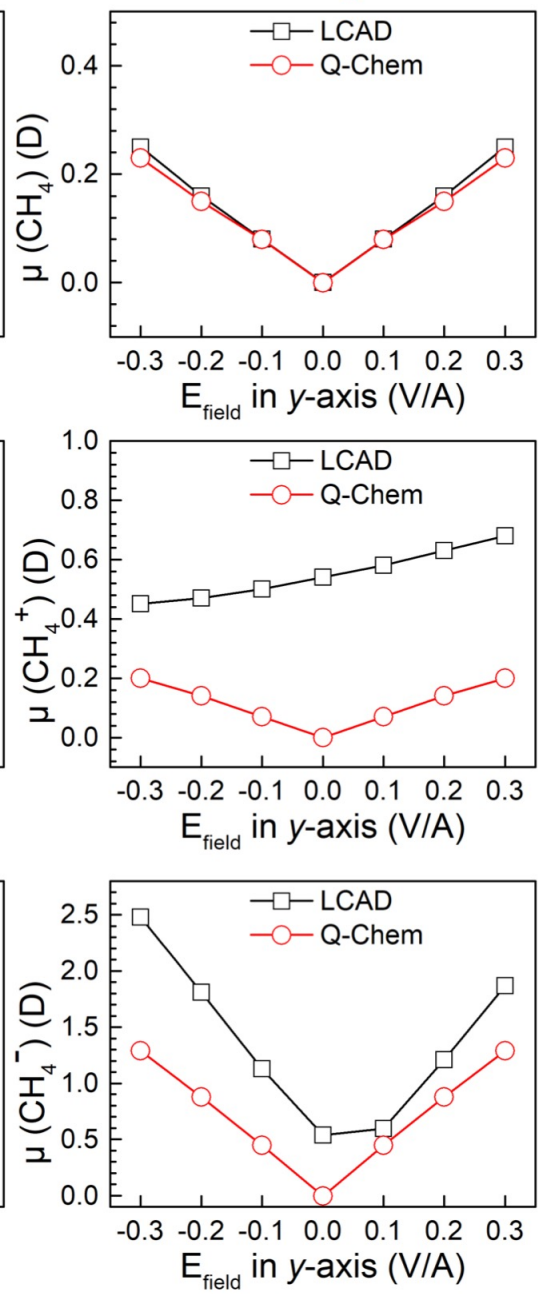
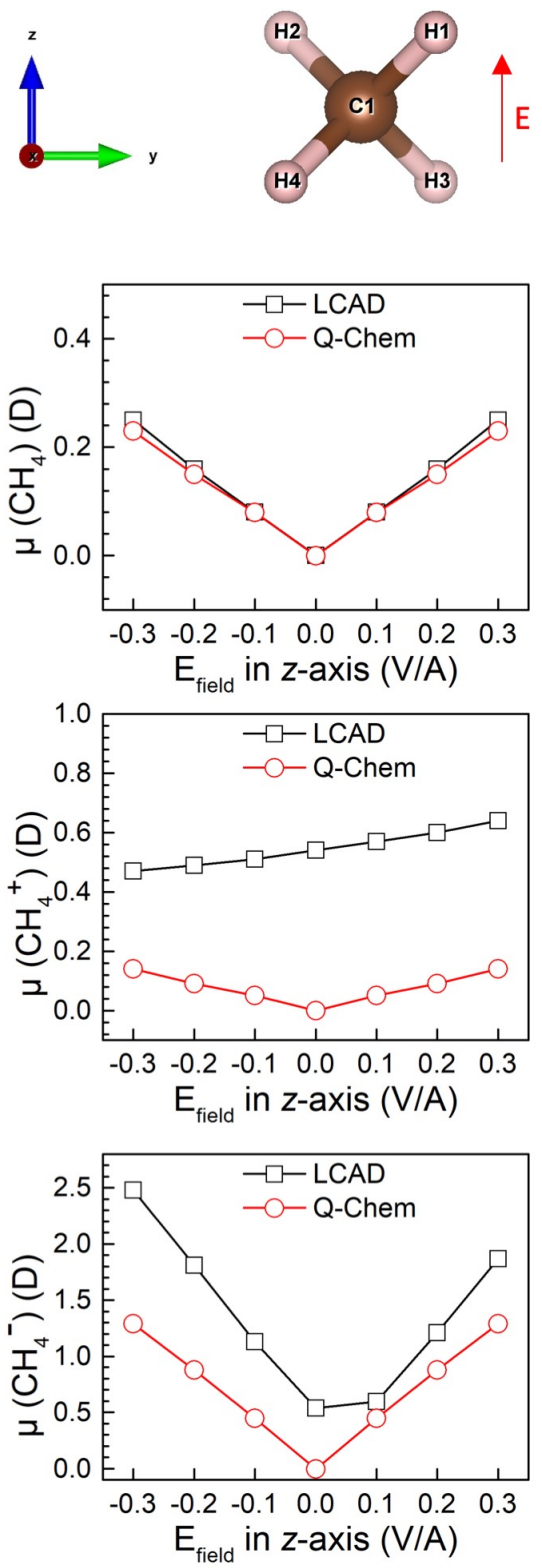

Figure S2: The molecular dipole moment in a) $\mathrm{CH}_{4}$ molecule, b) $\mathrm{CH}_{4}^{+}$cation and c) $\mathrm{CH}_{4}^{-}$anion calculated using LCAD method and Q-Chem under different uniform external electric fields.

Table S2: The bond dipoles of graphene flake $\mathrm{C}_{46} \mathrm{H}_{20}$ under zero and external electric field $\left(E_{y}=\right.$ $2.06 \mathrm{GV} / \mathrm{m})$. The unit of dipole moment and angle is Debye and ${ }^{\circ}$, respectively.

\begin{tabular}{ccccccc}
\hline Bond & Atom1 & Atom2 & Dipole & Angle $^{a}$ & Dipole $\left(\mathbf{E}_{y}\right)$ & Angle $\left(\mathbf{E}_{y}\right)$ \\
\hline C-C & C1 & C2 & 0.53 & 47.66 & 0.32 & 48.34 \\
& C1 & C5 & 0.06 & 22.88 & 0.20 & 82.11 \\
& C2 & C3 & 0.03 & 71.03 & 0.61 & 122.16 \\
\hline
\end{tabular}


Table S2 - continued from previous page

\begin{tabular}{|c|c|c|c|c|c|c|}
\hline Bond & Atom1 & Atom2 & Dipole & Angle & Dipole $\left(\mathbf{E}_{y}\right)$ & Angle $\left(\mathbf{E}_{y}\right)$ \\
\hline & $\mathrm{C} 2$ & C6 & 0.02 & 129.79 & 0.11 & 176.67 \\
\hline & $\mathrm{C} 3$ & $\mathrm{C} 4$ & 0.39 & 85.14 & 1.07 & 72.86 \\
\hline & $\mathrm{C} 4$ & C8 & 0.30 & 26.74 & 0.21 & 29.94 \\
\hline & $\mathrm{C} 5$ & $\mathrm{C} 10$ & 0.37 & 49.93 & 0.61 & 39.65 \\
\hline & C6 & C7 & 0.13 & 121.68 & 0.18 & 124.23 \\
\hline & C6 & C11 & 0.10 & 117.93 & 0.12 & 126.30 \\
\hline & C7 & $\mathrm{C} 8$ & 0.15 & 51.91 & 0.21 & 43.34 \\
\hline & $\mathrm{C} 7$ & $\mathrm{C} 12$ & 0.09 & 22.19 & 0.08 & 19.93 \\
\hline & $\mathrm{C} 8$ & C9 & 0.37 & 104.84 & 0.25 & 118.77 \\
\hline & C9 & $\mathrm{C} 14$ & 0.62 & 46.95 & 0.58 & 47.86 \\
\hline & $\mathrm{C} 10$ & C11 & 0.92 & 25.35 & 0.91 & 26.22 \\
\hline & $\mathrm{C} 11$ & C16 & 0.05 & 94.63 & 0.03 & 87.00 \\
\hline & $\mathrm{C} 12$ & C13 & 0.09 & 39.45 & 0.07 & 58.00 \\
\hline & $\mathrm{C} 12$ & $\mathrm{C} 17$ & 0.09 & 29.04 & 0.07 & 23.45 \\
\hline & $\mathrm{C} 13$ & C14 & 0.10 & 69.99 & 0.09 & 84.47 \\
\hline & $\mathrm{C} 13$ & C18 & 0.03 & 98.04 & 0.02 & 62.86 \\
\hline & C15 & C16 & 0.12 & 70.52 & 0.20 & 72.86 \\
\hline & C15 & $\mathrm{C} 20$ & 0.21 & 61.43 & 0.22 & 57.16 \\
\hline & C16 & $\mathrm{C} 17$ & 0.19 & 136.40 & 0.27 & 143.19 \\
\hline & $\mathrm{C} 17$ & $\mathrm{C} 22$ & 0.03 & 76.58 & 0.04 & 38.72 \\
\hline & $\mathrm{C} 18$ & C19 & 0.21 & 58.93 & 0.18 & 45.76 \\
\hline & C18 & $\mathrm{C} 23$ & 0.19 & 43.52 & 0.16 & 54.33 \\
\hline & C19 & $\mathrm{C} 24$ & 0.45 & 89.28 & 0.44 & 89.26 \\
\hline & $\mathrm{C} 20$ & C21 & 0.14 & 48.82 & 0.16 & 38.06 \\
\hline & $\mathrm{C} 21$ & $\mathrm{C} 22$ & 0.19 & 171.95 & 0.16 & 153.52 \\
\hline & $\mathrm{C} 21$ & C26 & 0.16 & 87.41 & 0.20 & 76.31 \\
\hline & $\mathrm{C} 22$ & $\mathrm{C} 23$ & 0.13 & 19.61 & 0.12 & 19.88 \\
\hline
\end{tabular}


Table S2 - continued from previous page

\begin{tabular}{|c|c|c|c|c|c|c|}
\hline Bond & Atom1 & Atom2 & Dipole & Angle & Dipole $\left(\mathbf{E}_{y}\right)$ & Angle $\left(\mathbf{E}_{y}\right)$ \\
\hline & $\mathrm{C} 23$ & $\mathrm{C} 28$ & 0.03 & 78.31 & 0.03 & 60.07 \\
\hline & $\mathrm{C} 24$ & $\mathrm{C} 29$ & 0.75 & 46.41 & 0.79 & 47.74 \\
\hline & $\mathrm{C} 25$ & $\mathrm{C} 26$ & 0.49 & 17.05 & 0.46 & 17.42 \\
\hline & $\mathrm{C} 25$ & $\mathrm{C} 30$ & 0.21 & 117.89 & 0.22 & 118.46 \\
\hline & $\mathrm{C} 26$ & $\mathrm{C} 27$ & 0.31 & 50.22 & 0.29 & 51.95 \\
\hline & $\mathrm{C} 27$ & $\mathrm{C} 28$ & 0.16 & 113.79 & 0.16 & 110.52 \\
\hline & $\mathrm{C} 27$ & $\mathrm{C} 32$ & 0.03 & 116.98 & 0.03 & 104.55 \\
\hline & $\mathrm{C} 28$ & $\mathrm{C} 29$ & 0.32 & 176.53 & 0.36 & 176.60 \\
\hline & $\mathrm{C} 29$ & $\mathrm{C} 34$ & 0.03 & 82.04 & 0.04 & 35.53 \\
\hline & $\mathrm{C} 30$ & $\mathrm{C} 31$ & 0.12 & 71.25 & 0.03 & 26.63 \\
\hline & $\mathrm{C} 31$ & $\mathrm{C} 32$ & 0.18 & 137.29 & 0.11 & 129.34 \\
\hline & C31 & $\mathrm{C} 37$ & 0.05 & 86.79 & 0.06 & 45.12 \\
\hline & C32 & $\mathrm{C} 33$ & 0.09 & 150.65 & 0.11 & 149.84 \\
\hline & $\mathrm{C} 33$ & $\mathrm{C} 34$ & 0.09 & 36.55 & 0.13 & 43.01 \\
\hline & $\mathrm{C} 33$ & $\mathrm{C} 39$ & 0.08 & 157.45 & 0.09 & 156.76 \\
\hline & $\mathrm{C} 34$ & $\mathrm{C} 35$ & 0.10 & 70.64 & 0.13 & 57.47 \\
\hline & $\mathrm{C} 35$ & $\mathrm{C} 41$ & 0.61 & 132.30 & 0.67 & 133.09 \\
\hline & $\mathrm{C} 36$ & $\mathrm{C} 37$ & 0.90 & 24.73 & 0.90 & 23.32 \\
\hline & C36 & $\mathrm{C} 42$ & 0.36 & 129.46 & 0.43 & 133.41 \\
\hline & $\mathrm{C} 37$ & $\mathrm{C} 38$ & 0.10 & 60.83 & 0.06 & 52.03 \\
\hline & $\mathrm{C} 38$ & C39 & 0.12 & 121.71 & 0.06 & 108.36 \\
\hline & C38 & $\mathrm{C} 44$ & 0.02 & 49.89 & 0.09 & 161.22 \\
\hline & C39 & C40 & 0.14 & 51.65 & 0.06 & 78.79 \\
\hline & $\mathrm{C} 40$ & C41 & 0.36 & 105.51 & 0.51 & 98.29 \\
\hline & $\mathrm{C} 40$ & C46 & 0.29 & 153.08 & 0.43 & 155.39 \\
\hline & $\mathrm{C} 42$ & $\mathrm{C} 43$ & 0.07 & 155.93 & 0.26 & 121.04 \\
\hline & $\mathrm{C} 43$ & $\mathrm{C} 44$ & 0.52 & 47.49 & 0.78 & 47.54 \\
\hline
\end{tabular}


Table S2 - continued from previous page

\begin{tabular}{ccccccc}
\hline Bond & Atom1 & Atom2 & Dipole & Angle & Dipole $\left(\mathbf{E}_{y}\right)$ & Angle $\left(\mathbf{E}_{y}\right)$ \\
\hline & C44 & C45 & 0.03 & 71.03 & 0.15 & 116.92 \\
& C45 & C46 & 0.39 & 85.45 & 0.53 & 79.98 \\
\hline C-H & C1 & H6 & 0.09 & 57.47 & 1.59 & 13.75 \\
& C3 & H1 & 0.73 & 176.51 & 2.34 & 6.35 \\
C4 & H2 & 0.54 & 71.96 & 1.21 & 53.51 \\
C5 & H7 & 0.70 & 58.27 & 2.34 & 41.61 \\
C9 & H15 & 0.25 & 74.83 & 0.72 & 39.37 \\
C10 & H8 & 0.30 & 155.48 & 0.50 & 123.27 \\
C14 & H16 & 0.55 & 109.35 & 0.55 & 111.25 \\
C15 & H9 & 0.45 & 28.52 & 0.66 & 23.61 \\
C19 & H17 & 0.66 & 31.78 & 0.76 & 28.41 \\
C20 & H10 & 0.50 & 147.17 & 0.71 & 149.98 \\
C24 & H18 & 0.69 & 156.48 & 0.82 & 158.62 \\
C25 & H11 & 0.71 & 149.46 & 0.46 & 147.54 \\
C30 & H12 & 0.43 & 26.70 & 0.25 & 36.95 \\
C35 & H19 & 0.54 & 109.09 & 0.52 & 108.95 \\
C36 & H13 & 0.30 & 156.04 & 0.33 & 149.25 \\
C41 & H20 & 0.24 & 75.44 & 0.37 & 172.58 \\
C42 & H14 & 0.68 & 58.24 & 0.46 & 174.90 \\
C43 & H4 & 0.08 & 61.58 & 1.43 & 171.07 \\
C45 & H5 & 0.72 & 176.48 & 2.16 & 175.11 \\
H3 & 0.52 & 72.23 & 0.38 & 171.81 \\
\hline
\end{tabular}

${ }^{a}$ The angle value denotes the orientation difference between the bond direction and its calculated bond dipole. 


\section{References}

(1) Lu, T.; Chen, F. Atomic dipole moment corrected Hirshfeld population method. J. Theor. Comput. Chem. 2012, 11, 163-183.

(2) Cho, M.; Sylvetsky, N.; Eshafi, S.; Santra, G.; Efremenko, I.; Martin, J. M. The Atomic Partial Charges Arboretum: Trying to See the Forest for the Trees. ChemPhysChem 2020, 21, 688696. 